Supporting Information for

\title{
Three-Dimensional Optical Anisotropy of Low-Symmetry Layered GeS
}

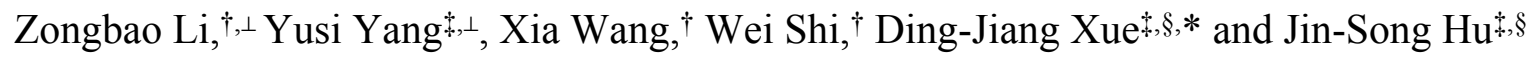

†School of Material and Chemical Engineering, Tongren University, Tongren 554300, China

\#eijing National Laboratory for Molecular Sciences (BNLMS), CAS Key Laboratory of Molecular Nanostructure and Nanotechnology, Institute of Chemistry, Chinese Academy of Sciences (CAS), Beijing 100190, China

§University of Chinese Academy of Sciences, Beijing 100049, China

${ }^{\perp}$ These authors contributed equally.

* To whom correspondence should be addressed. E-mail: djxue@iccas.ac.cn

This file includes Figure S1-S9. 
(a)

(b)

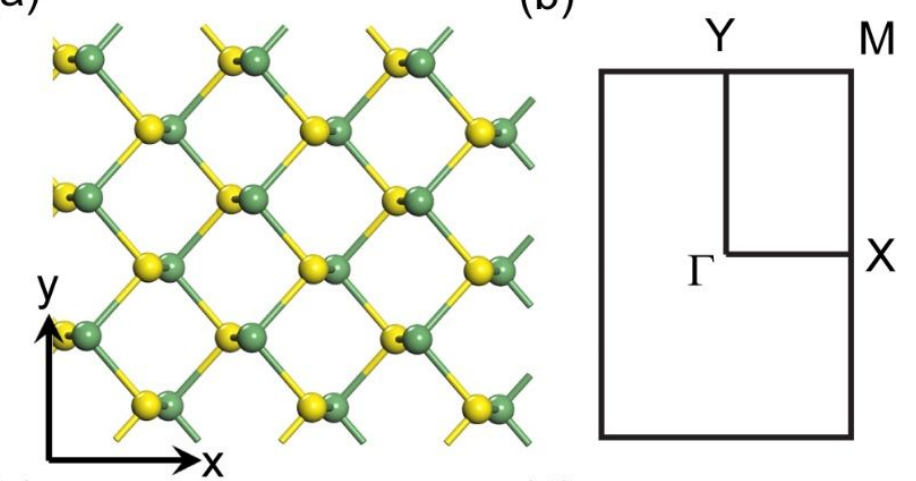

(c)

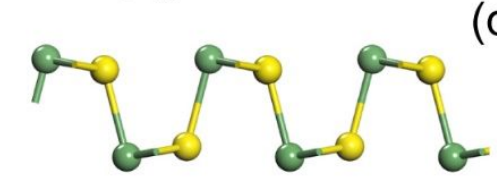

(d)
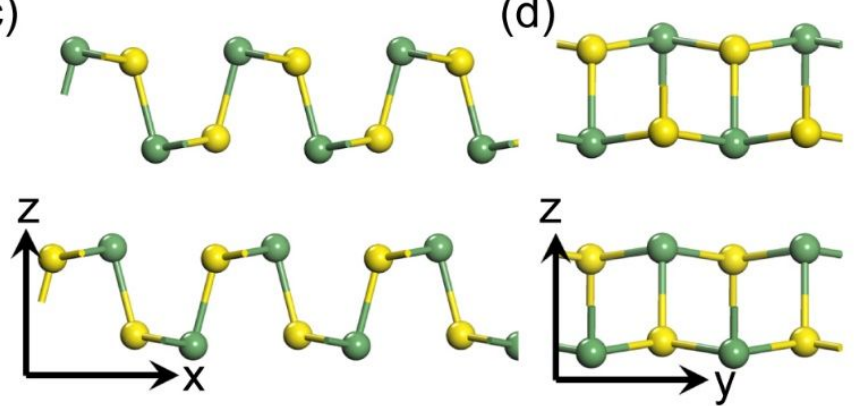

Figure S1. (a) Top view of GeS. (b) Schematic of 2D Brillouin zone of GeS. (c)-(d) Side views of GeS for $\mathrm{x}-\mathrm{z}$ and $\mathrm{y}-\mathrm{z}$ planes. 


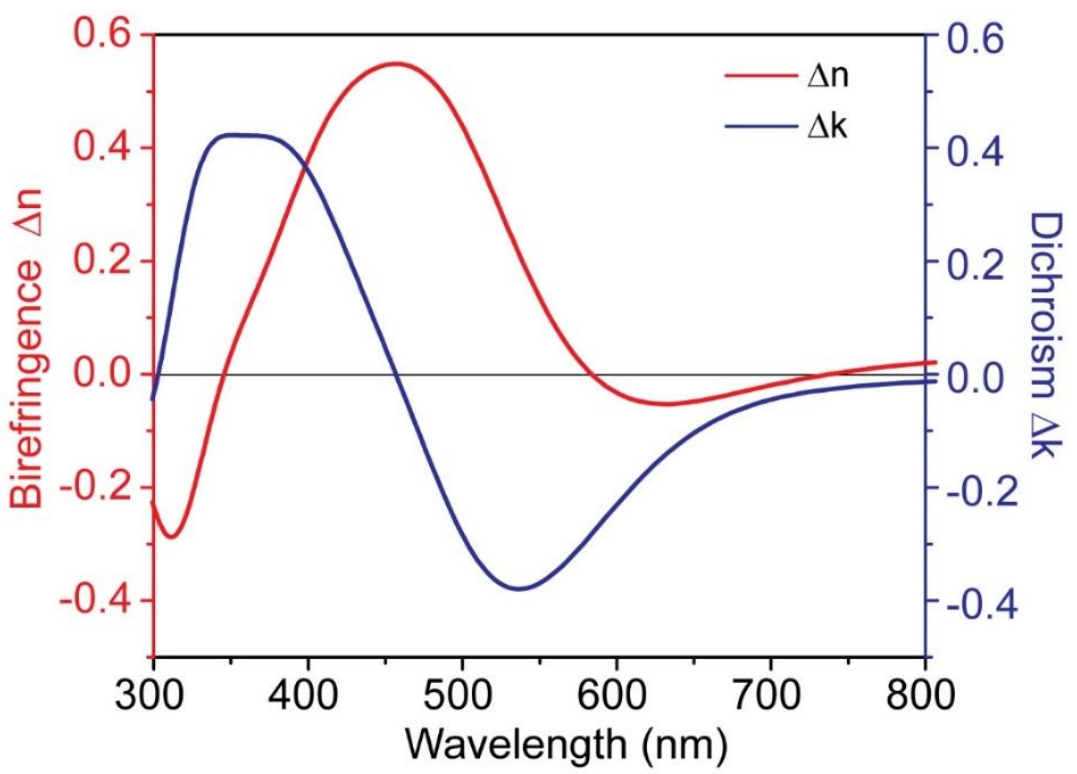

Figure S2. Calculated birefringence $(\Delta \mathrm{n})$ and linear dichroism $(\Delta \mathrm{k})$ for wavelength from 300 $800 \mathrm{~nm}$. 


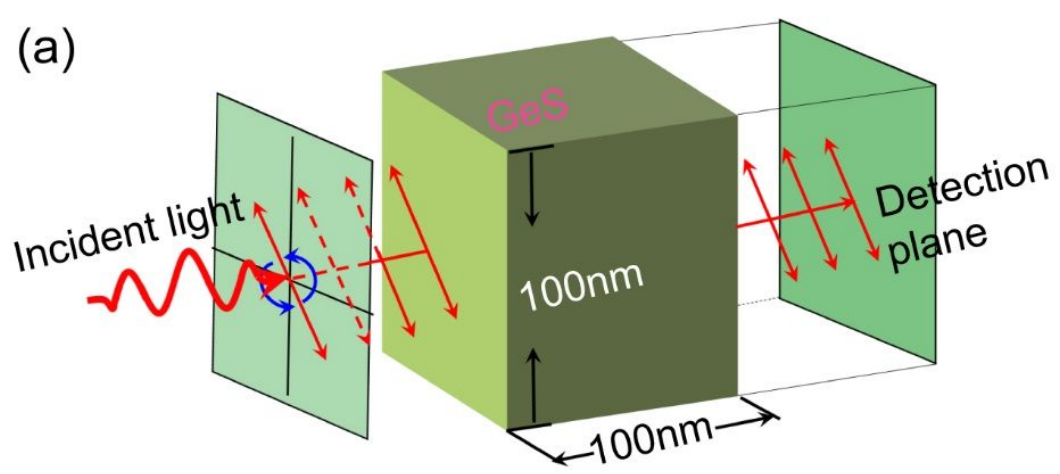

(b)

(c)

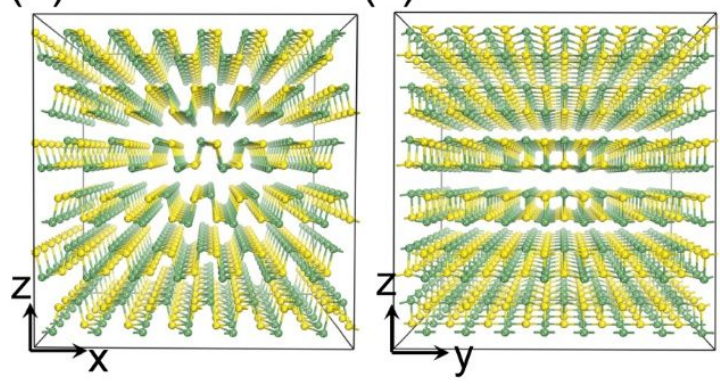

(d)

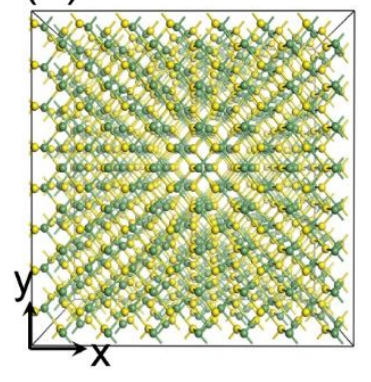

Figure S3. (a) Schematic diagram of absorbance along one direction. (b)-(d) Side view of three light incident planes of GeS flakes. 


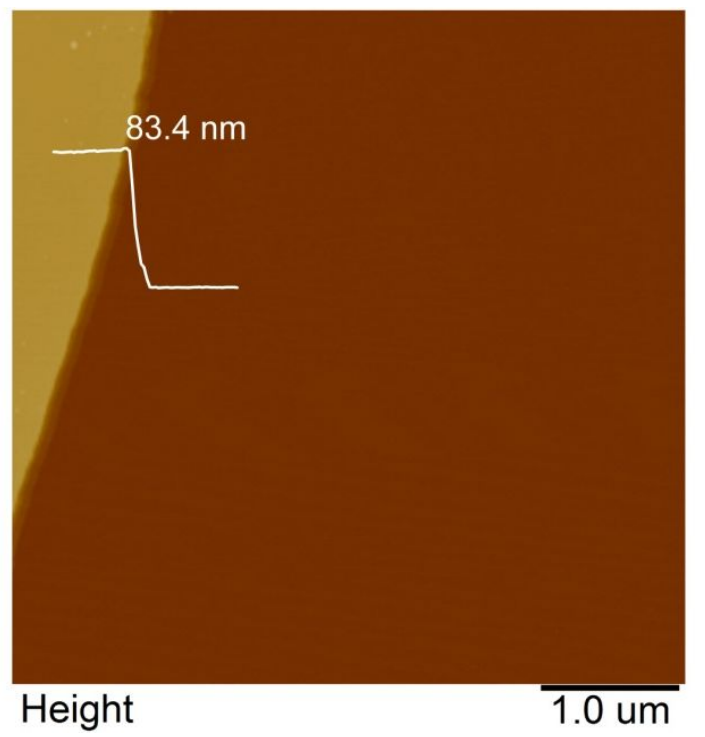

Figure S4. AFM images and profiles of GeS flake in testing of PRAS. 


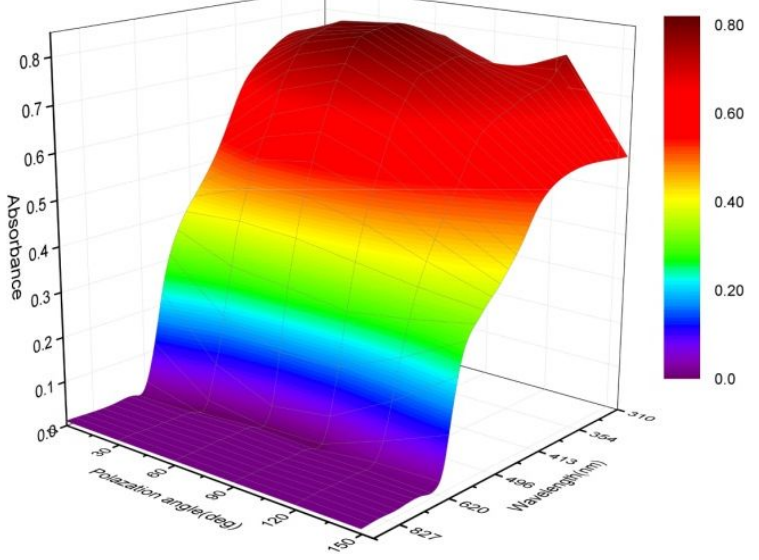

Figure S5. 3D view of polarization-resolved absorption spectra of GeS flake. 
(a)

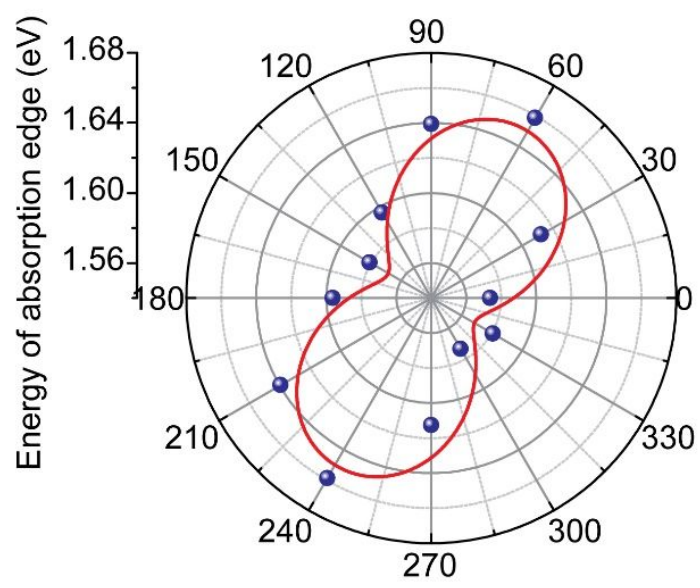

(b)

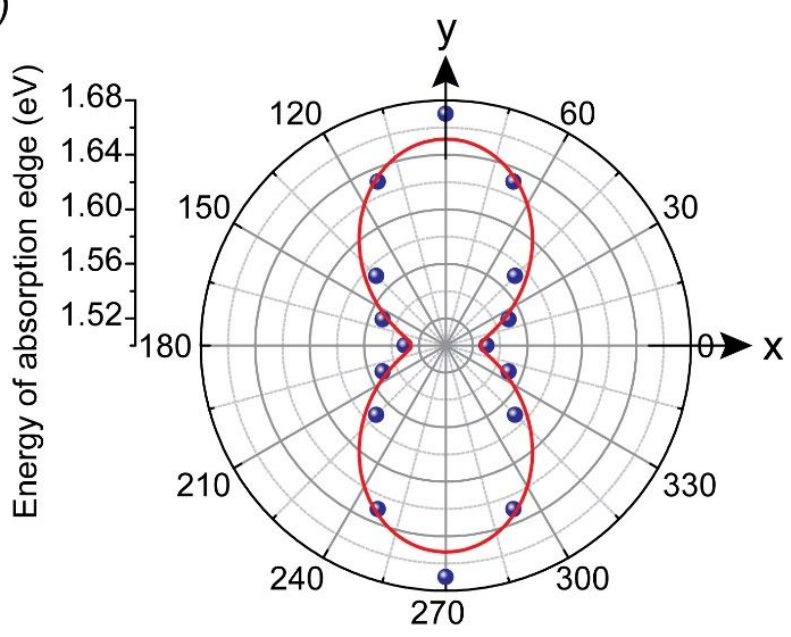

Figure S6. Evolution of the energy of absorption edges in (a) experiment and (b) DFT calculation plotted in the polar coordination. 


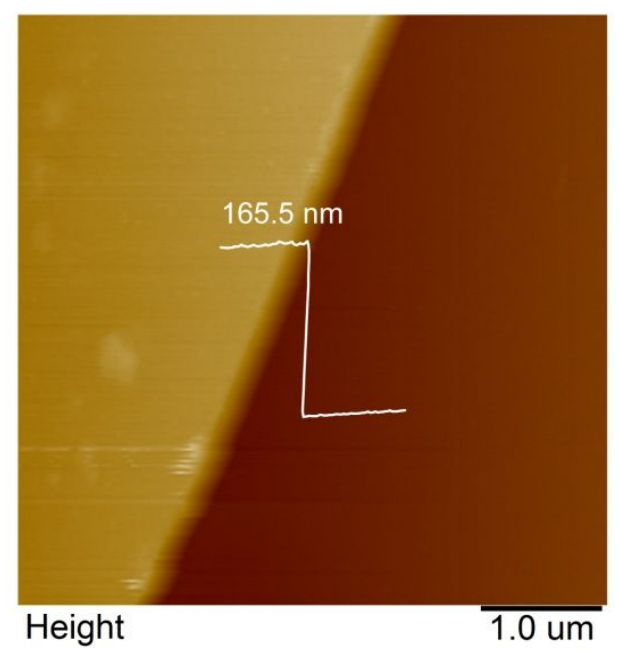

Figure S7. AFM image and profile of GeS flake in ARPRS and PROM measurements. 
(a)

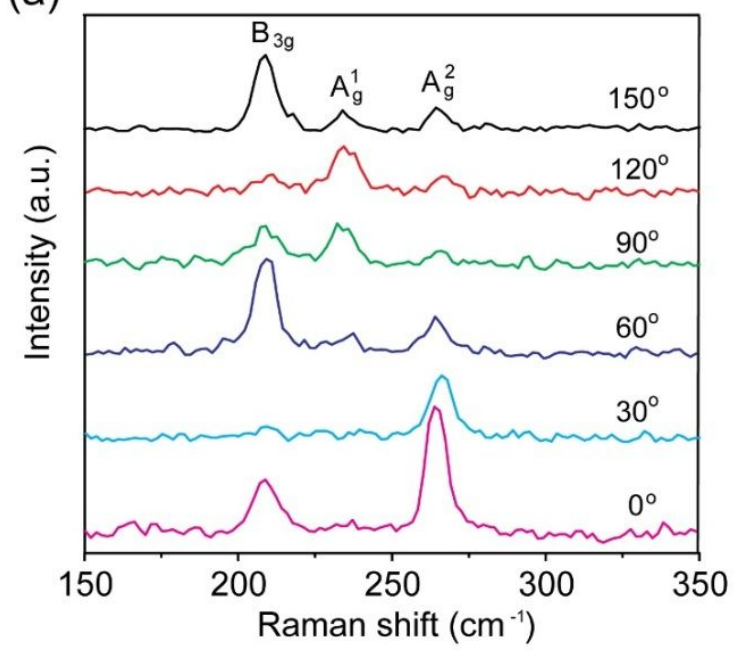

(b)

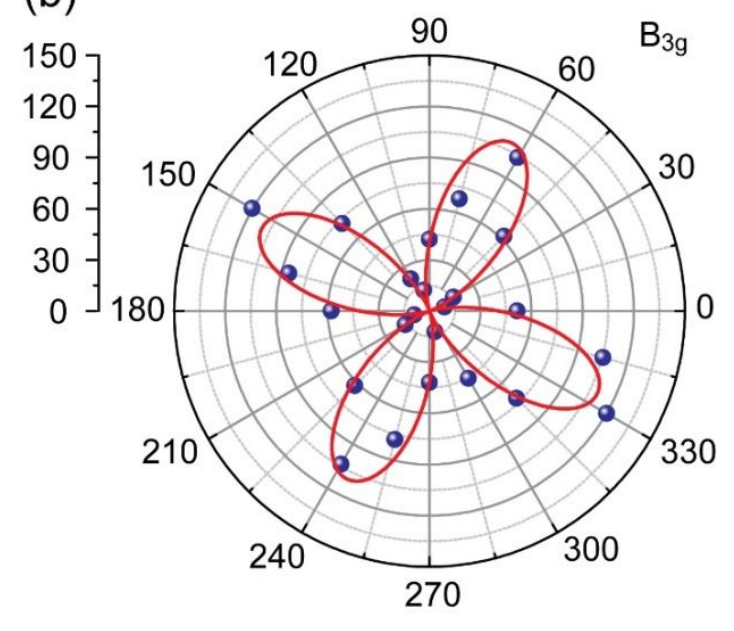

Figure S8. (a) Angle-resolved polarized Raman spectra of GeS flake under parallel configuration. (b) Polar plots of the Raman-peak intensities of the $\mathrm{B}_{3 \mathrm{~g}}$ mode. 


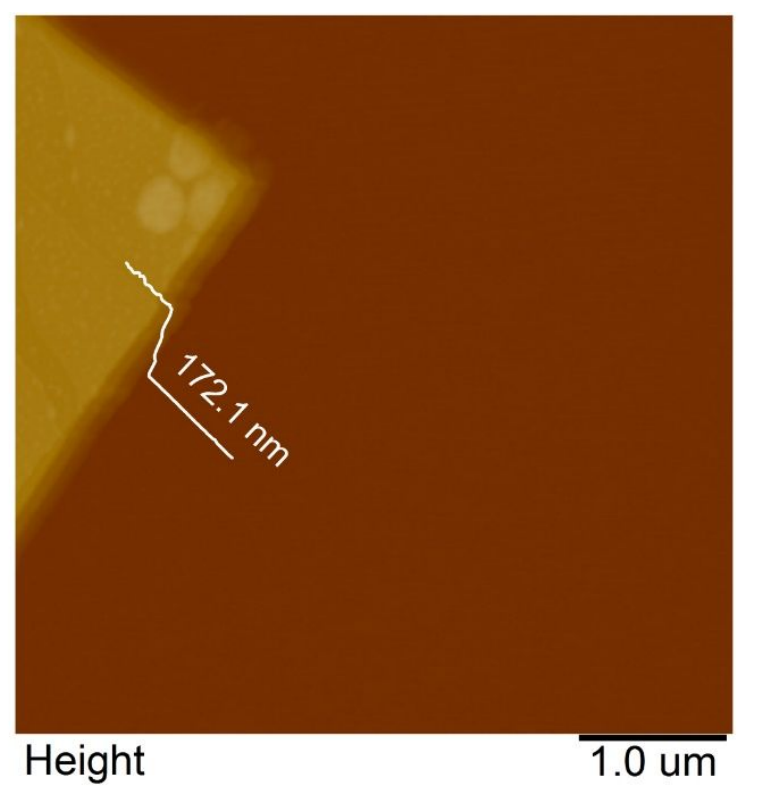

Figure S9. AFM image and profile of GeS flake in testing of ADRDM. 